ein Drittheil unterirdisch, und breitele sich oben anf 22 Cm. aus. Oher- und Unterseite der Hüte, Farbe, Fleischbeschaffenheit, kurz alles bis auf die Form war normal.

\title{
Plantas in itinere africano
}

\section{ah J. M. Hildebrandt collectas determinare pergit W. Vatke.}

\section{Composita e Vaill.}

1013. Ethulia conyzoides L: var. angustifolia (Bojer.). In planitiebus secus ripas fluminis Kingani in Sansibariae ore aug. 1873.

Suffrutex altit. $1 \mathrm{~m}$. fide $H$., sed annua, ut jam DC. indicavit, colitur in horto berol., ubi olim a cl. Regel $E$. corymbosae nomen, a cl. Steetz in Peters Mosamb. Bot. p. 322 commemoralum accepit.

403. Vernonia cinerea (L.) Less. Abyssinia: Habab ad alt. $3000^{\circ}$ junio 1872.

1016. eadem. In Sansibariae campis cultis et pratis siccis herbaceis jul. 1873 ; fl. lilacini.

412. V. cyanopidea Hochst. in Schweinf. Beitr. p. 161, ( $V$. Aschersonii C. H. Schultz bip. ibidem). Abyssinia: Habab inter saxa granitica et lapides schistosos sept. 1872. Nomen prius, 1. c. rite editum, rejectum est ob. $V$. cyanopioidem Walp., quae vero ex Benth. FI. austr. III. p. 459 eadem quae $V$. cinerea (L.) Less. Itaque nunc nomen hochstetterianum restituendum esse censeo.

768. V. spathulata (Forsk.) C. H. Schultz. bip. in Schweinf. Beitr. p. 162. ( $V$. atriplicifolia Jaub. et Spach.) Aden ad montes jun. 1872 maxima ex parte jam deflorata; frutex $1 \mathrm{~m}$. altus. mart. 1873.

843. eadem. In Somalensium montibus $A$ hl alt. $1000 \mathrm{~m}$. vulgaris

1020. V. (Lepidaploa) Hildebrandtii Vatke. canle fruticoso tereti striato hispidulo, foliis petiolatis subovato-lanceolatis crenatis obtusis; basi utrinque auriculatis supra parce, subtus dense hispidulis, corymbo polycephalo, capitulis sub 10-floris, involucri squamis sub 3-serialibus ovato-lanceolatis obtusis-pilosulis, achaeniis sub 万-costatis, secus costas puberulis, pappi albi serie exteriore minuta setiformi, interiore 1 -seriali multiseta $\overline{5}$.

Insula Sansibar nov. 1873. Frutex densus (H.) $2 \mathrm{~m}$. altus ex affinitate $V$. capensis (Spr. f) Vatlie ined. (V. mespilifoliae Less.); petioli c. $1 \mathrm{~cm}$. Iongi; lamina c. $4 \mathrm{~cm}$. $\lg$., ad $2 \mathrm{~cm}$. lata, subtus reticulatomenosa; achaenia nunc manifeste costata et ad costas puberula, nunc costis obliterantibus ubique pilis tecta. Species distinctissima peregrinatoris nostri indefessi nomen servet. 1873.

1019. V. zanzibarensis Less.! In Sansibariae locis siccis jun. 
405̆. V. pauciflora (Willı.) Less. Abyssinia: Habab alt. 3$6000^{\prime}$ sept. 1872 forma exilis.

413. V. polymorpha Vatke $\gamma$ accedens Vatke pl. Schimper. Abyssinia: Habab Nakfa alt. $5000^{\prime}$ aug, 1872 ; frutex usque ad 2 m. altus; fl. violacei.

1014. V. (Lepidella) poskeana Vatke et Hildebrandt. caule erecto herbaceo divaricato-ramosissimo striato puberulo, foliis lineari-oblongis obtusis utrinque supra dense puberulis, subtus punctulatis, margine recurvulis subintegerrimis, corymbis polycephalis, involucri squamis pilosis, exterioribus ovato-lanceolatis, interioribus lanceolato-linearibus acutis mucronatis apice medio coloratis flores subaequantibus, achaeniis 5-striatis pubescentibus, pappo albido, exteriore 10-paleaceo, interiore sub 15 -seto caducissimo. $\odot$.

Sansibar in pratis siccis herbaceis jun. 1873; herba altit. $1 . \mathrm{m}$, folia ad $5 \mathrm{~cm}$. longa, ad $2 \mathrm{~cm}$. lata; pappus exterior persistere videtur.

Speciem insignem dicavimus Friederico Poske viro ingenioso, studiorum olim socio amicissimo.

1011. Ageratum conyzoides $\mathbf{L}$. In pratis herbaceis et campis cultis Sansibariae jun. ad nov. 1873.

1017. Mikania chenopodiifolia Willd. (M. mossambiquensis Steetz in Peters Mosamb.) In silvis secus ripas fluminis Kingani in Sansibariae ore suffrutex volubilis aug. 1873 .

407. Dichrocephala ehrysanthemifolia (BI.) DC. var. abyssinica (C. H. Schultz bip.) Aschs. in Schweinf. Beitr. Abyssinia: Habab alt. 6-7000' aug. 1872.

399. Felicia abyssinica C. H. Schultz. bip., A. Rich. tent. Abyssinia: Habab Nakfa, Rora, aug. 1872; fl. radii violacei.

411. Conyza macrorhiza C. H. Schultz bip., A. Rich. tent. Abyssinia: Habab in planitiebus siccis aug. ad sept. 1872.

409. Psiadia arabica Jaub. el Spach. (? P. resiniflua C. H. Schultz in Schweinf. Beitr. nomen.) Abyssinia: Ilabab in solo granit. alt. $6000^{\circ}$ jul. ad aug. 1872 ; frutex $2 \mathrm{~m}$. altus.

6976. eadem etiam ex Abyssinia. Eandem ibidem prope Keren. c. 4500 p. jul. 1870 . coll. Beccari! (n, 214).

672. Tarchonanthus camphoralus L. (? T. abyssinicus C. H. Sch. b. in Schweinf. Beitr. nomen). Abyssinia: Habab: Nakfa aug. 1872; arbor $4 \mathrm{~m}$. alta ramis laxis; forma latifolia.

883. idem. E. Somalensium montibus Ahl ad Yafir alt. $2000 \mathrm{~m}$. mart. 1873 ; frutex $3 \mathrm{~m}$. altus.

A stirpe capensi admodum variabili vulgo in hortis bot. culta nostram equidem distinguere nequeo.

1012. Blumea lacera (Burm.) DC. Kokotoni Sansibariae in declivibus collium calcareorum e corallis ortorum oct. nov. 1873; suffrutex (H.) potius herba $0.5 \mathrm{~m}$. alta.

4036. B. Bovei (DC.) Vatke ined. (Conyza B. DC., B. abyssinica C. H. Sch. bip., A. Rich. tent.) Abyssinia: Habab ad alt. $3000^{\prime}$ jun. 1872. 
1022. Laggera sordida Vatke. fruticosa ramosissima, ramis fuscis elevato-striatis superne pilosis, foliis obovato-oblongis obtusis in petiolum anguslatis integerrimis, capitulis numerosis parvis breviter pedunculatis in paniculas confertas ramulos terminantes dispositis, involucri squamis imbricatis, exterioribus ovato-lanceolatis, interioribus linearibus acutis pilosulis, floribus $\mathbf{Q}$ pluriserialibus, corolla tubulosa filiformi apice 2 dentata stigmatibus exsertis, in floribus $\overline{0}$ paucis $Q$ superantibus stylo profunde 2-lobo, pappi 1-plicis setis scabris, acliaeniis adpresse setosis. $\zeta$.

In Insulae Sansibar locis lapidosis ab aestu maris interdum humefactis oct. 1873 .

Frutex altit. $1 \mathrm{~m}$. aequat; folia petiolo incl. ad $2 \mathrm{~cm}$. longa, ad $0.5 \mathrm{~cm}$. lata; antherae basi sagittatae more generis.

404. Laggera aurita (L.) C. H. Schultz bip. Abyssinia: Habab ad altit. $3000^{\prime}$ jun. 1872 .

406. eadem ibidem lecta forma lignosa.

410. Pluchea Dioscoridis (L.) DC. Abyssinia: Habab alt. $3-$ 7000 aug. 1872; frutex $2 \mathrm{~m}$. altus. Eandem prope Keren (Bogos) maio 1870 coll. Beccari! (n. 67).

765. P. indica (L.) Less. var. Schech Othman prope Aden in littore arenoso jun. 1872. Habitus plantae summitatibus decerptis paullulum mutatus, sed procul dubio buc refero.

1018. P. Kraussii C. H. Schultz bip. in Walp. rep. II. p. 972 (sub Conyza), Steetz in Peters Mosamb. var. foliis integerrimis. Insula Sansibar in locis paludosis prope mare jun. ad nov. 1873; frutex $2 \mathrm{~m}$. altus; flores lilacini.

Speciei hujusce praesto mihi sunt solummodo exempl. orig. varietatum duarum a cl. Steetz in Peters Mosamb. Bot. p. 442 descriptarum, quarum cum nulla convenit, sed species sane eadem.

842 a. P. (Oligocephalum) heterophylla Vatke. basi frutescens superne ramosa, foliis obovato-oblongis, in petiolum angustalis, nunc integerrimis obtusiusculis, nunc profunde inciso-dentatis, lobis apiceque acutis, utrinque lepidoto-punctatis, capitulis paucis pedunculatis subcorymbosis, involucri squamis pluriserialibus angustis apice recurvis. 5 .

In calcareis montium All d. alt. 1-2000 m. mart. 1873 copiose, sed maxima ex parte nondum florens.

Fruticulus humilis parum ramosus c. $2 \mathrm{dm}$. altus superne oum involucri squamis glandulis stipitatis obtectus ceterum glaber ramulis tenuibus foliosis; folia carnosula? ad $4 \mathrm{~cm}$. Ionga, ad $0.5 \mathrm{~cm}$. lata; pedunculi bracteati; involucrum hemisphaericum diam. superne c. 2 cm., squamis exterioribus gradalim brevioribus obtusiusculis, intimis acutis, receptaculo breviter foveolato; flores $\mathbf{Q}$ multiseriati numerosi corolla tubulosa filiformi apice 2-dentala, sligmatibus exsertis, flores $\sigma$ in medio capitulo numerosi $O$ superantes corolla anguste lubulosa apice breviter 5 fida, stylo indiviso summo apice levissime 2-lobo; antherae basi breviter caudato-acuminatae; achaenia adpresse setosa; pappi simplicis setae scaberulae.

Oesterr. botan. Zeitschrift. 10, Heft, 1875. 
P. pinnatifidae Hook. I. in Hook. ic. pl. t. $115 \mathrm{5} 6$ ex eadem re-m gione proxima, sed abunde distincta.

1021. Sphaeranthus microcephalus Willd. In Sansibariae locis paludosis oct. 1873.

14. Filago pyramidata L. fide C. H. Schultz bip.! (F. spathulata Presl.). In deserto Ramle prope Alexandriam mart. 1872. tempore.

13. Ifloga spicata (Forsk.) C. H. Schultz bip. Ibidem eodem

420. Achyrocline lazuloides (C. H. Schultz bip.) Vatke ined. (Gnaphalium 1. Sch. bip. in Schweinf. Beitr. p. 149). Abyssinia: Habab alt. $5-7000^{\prime}$ in solo granitico jul. ad sept. 1872; fruticulus alt. 0.5 m. Eandem prope Keren repperit Beccari! jul. 1870 (n. 211).

841 b. Inula somalensis Vaike. caule fruticoso erecto ramoso, ramis lignosis angulatis superne pubescentibus, foliis alternis obovatooblongis, basi dilalata subauriculata subsemiamplexicaulibus antice denticulatis, dentibus apiceque in mucronem productis subtus punctatis, margine costaque infera longe pubescenti-villosis, capitulis breviter pedunculatis solitariis radiantibus, involucri squamis obovato-lanceolatis laxe imbricatis mucronatis subaequilongis. accessoriis paucis, achaeniis teretibus a basi ad apicem adpresse setosis. 5 .

In solo calcareo montium AhI d. mart. 1873 sp. unicum. Fruticulus alt. $2 \mathrm{dm}$. habitu Pulicariae; folia ad $5 \mathrm{~cm}$. longa, ad $1 \mathrm{~cm}$. lata; receptaculum areolatum; pappi setae 15 barbellatae.

767. Iphiona scabra D.C. Áden ad montes jun. 1872 frutex 1 m. allus ramificatione densa.

67. Pulicaria undulata (L.) D C. Inter Trifolia culta prope Suez apr. 1872 .

405 b. ejusdem var. Abyssinia: Habab: Keren aug. 1872.

128 ejusdem var. ( $P$. orientalis Jaub. et Spach.) Geddah apr. 1872.

842. P. (Decachaeta) Hildebrandtii Vatke. suffruticosa in partibus herbaceis pubescenti-pilosa, foliis late obovatis ohtusis, basi dilalata amplexicaulibus ultra medium ab apice grosse inaequaliter incisodentatis, dentibus apiceque mucronatis, pedunculis terminalibus 1 -cephalis brevibus subnudis, capitulis subhemisphaericis radiatis, involucri squamis exterioribus lanceolatis herbaceis, interioribus linearilanceolatis scariosis laxe imbricatis, receptaculo alveolato, achaeniis teretibus 10 -costatis bis articulatis. $\zeta$.

In planitie littorali et promontoris montium Ahl ad $1000 \mathrm{~m}$. mart. 1873; nomen vernac. Farre odd. ut n. 841 (probabil. Inula somalensis) et complures Compositae, ut Somalenses saepius stirpes similes nomine generali salutant.

Suffrutex $0.5 \mathrm{~m}$. altus, habitu $P$. grandidentatae Jaub. et Spach simillimus, sed involucium non convenit. Folia odoratissima ad $6 \mathrm{~cm}$. longa, ad $3 \mathrm{~cm}$. lata; achaenia forte monstruosa? pappi exterioris dentes breves parum laceri, interioris setae ad 20 barbellatae, quo quidem numero cum definitione subgeneris a cl. Spach data non congruit, sed reliqua omnia conveniunt. 
766. P. glutinosa Jaub. et Spach. Aden ad montes jun. 1872.

$841=841$ a. $P$. (Platychaeta) kurtziana Vatke. suffruticosa in partibus herbaceis dense glandulifera et parce pilosa, foliis oblongolinearibus apicem versus grosse pinnatifido-dentatis carnosulis? callosomucronulatis, pedunculis 1 -cephalis, capilulis multifloris radiatis, anthodii polyphylli late campanulati squamis ovato-sublanceolatis inaequilongis, exterioribus gradatim minoribus iisque apice fuscis acutiusculis glabriusculis carinatis, floribus radialibus disco vix triente longioribus, ligula obovato-oblonga 3-dentala, achaeniis oblongis 8-costulatis glabris, pappi externi campanulati leviter inciso-denticulati lobis acutiusculis, interiore corollae florum $Q$ subaequilongo. $\mathbf{5}$.

In calcareis montium Ahl ad alt, $1000 \mathrm{~m}$. mart. 1873.

Fruticulus $0.5 \mathrm{~m}$. altus praecedenti et P. Grantii Oliv: et Hiern. affinis; folia ad $4 \mathrm{~cm}$. longa, ad $1.5 \mathrm{~mm}$. lata; radius luteus; pappi setae 8 spathulatae latiusculae margine serrulatae, apice plumosobarbellatae; setarum numerus in hac quoque non congruit cum subgeneris definitione a cl. Spach in III. pl. or. 346 data, sed reliqua omnia conveniunt; stigmata exserta recta lineari-filiformia generis more papillosa; antherae lineares appendicibus elongatis laceris, omnino ut in praecedente a ell. Jaubert et Spach sunt depictae.

Species dicata Friederico Kurtz excursionis hildebrandtianae fautori mihique amicissimo.

68. P. (Francoeuria) crispa (Forsk.) Benth, et Hookf. Suez inter Trifolia culta apr. 1872. Adn. auctores quidam, si genus reduxere viri cll. Bentham et Hooker filius, etiam omnibus speciebus in novo genere hos adscribunt auctores, equidem tantum, si quo modo speciem commemorant, hos cito.

127 eadem. Geddah apr. 1872.

402 eadem. Abyssinia: in planitie Habab prope Keren jul. ad sept. 1872. Ibidem in valle Insaba, prope Keren coll. Beccari! maio 1870 (n. 59 ).

Obs. P. petiolaris Jaub. et Spach. est P. chrysopsidoides C. H. Schultz bip., de qua confer Schweinf. Beitr. p. 153.

419. Geigeria acaulis (C. H. Schultz bip.) Benth. et Hookf. (Diplostemma a Schultz). Abyssinia: Habab 5-6000' in planitiebus lapidosis tempore pluvioso aug. 1872. Novus florae abyssinicae civis.

65. Odontospermum graveolens (Forsk.) C. H. Schultz. bip. (Ceruana pratensis Aschs.! in Hildebr. pl. exs. non Forsh.). Suez inter Trifolia culta apr. 1872.

414. Sclerocarpus africanus Jacq. Abyssinia: Habab prope Keren tempore pluvioso sept. 1872 sub fruticetis.

418. Wedelia abyssinica Valke. (Wirtgenia frutescens C. H. Schultz bip. in Schweinf. Beitr. p. 287 nomen nudum).

Frutescens caule erecto superne ramoso tereti strigoso, foliis breviter petiolatis ovato- vel oblongo-lanceolatis acutis repando-subserratis supra scabris vel utrinque hirtis 3-nerviis, capitulis longe pedicellatis, ad apices ramorum 1-3, involucri squamis ovato-lanceolatis obtusiusculis strigoso-hirtis subbiserialibus, flosculis luteis, re- 
ceptaculi paleis oblongo-linearibus mucronato-acutis, achaeniis obovoideo-oblongis subcompressis strigoso-hirtis apice abrupte in collum breve desinentibus, pappo exteriore ex aristis 2 longioribus, interiore e squamellis pluribus composito. $\overline{5}$.

Abyssinia: Habab, Rora asgede rara aug. 1872.

Frutex $1 \mathrm{~m}$. altus; rami ad $4 \mathrm{dm}$. longi; folia $4-8 \mathrm{~cm}$. longa, $1-2 \mathrm{~cm}$. lata; flores radii aurantiaci feminei, quare Wedeliae nec Aspiliae species.

1015. Aspilia Kotschyi (C. H. Schultz bip.) Benth. et Hookf. (Wirtgenia Kotschyi Schultz). Insula Sansibar in locis udis rara oct. 1873. annua (videtur H.) procul dubio.

117. Coreopsis prestinaria C. H. Schultz bip., (Verbesina veris A. Rich. tent.). Abyssinia: Habab tempore pluvioso sept. 1872. 1873.

1023. Bidens pilosus L. In Sansibariae loco sterili oct. nov.

415. B. abyssinicus C. H. Schultz bip., A. Rich. tent. $\beta$ glabratus Vatke pl. Schimper. Abyssinia: Habab alt, 5000' tempore pluvioso sept. 1872.

Fere idem, qui in Schimp. pl. abyss. sect. III. n. 1427 editus, sect. I. n. 337 est forma typica.

Stirps quibus differat characteribus certis a $B$. bipinnato L. nondum intellexi.

416. Chrysanthellum indicum DC. Abyssinia: Habab alt. 5000 in solo granitico tempore pluvioso sept. 1872.

15. Anthemis retusa Del. In deserto Ramle prope Alexandriam mart. 1872.

64. eadem. Inter Trifolia culta prope Suez apr. 1872.

66. Cotula cinerea Del. cum praecedente.

1027. Gynura microcephala Vatke. Caule herbaceo erecto tereti parce puberulo striato, foliis late lanceolatis in petiolum angustatis a medio ad apicem inaequaliter repando-dentatis acutiusculis, corymbo densiuscule oligocephalo, pedicellis brevibus erectis demum cernuis, involucro sub 16-phyllo anguste campanulato flosculis subbreviore, bracteolis subulatis sub 5 plo longiore, achaeniis teretibus striatis ad costas puberulis. $\odot$.

In Sansivariae locis cultis oct. ad nov. 1873.

Herba altit. 0.5 m.; folia ad $1 \mathrm{dm}$. Ionga, ad $4 \mathrm{~cm}$. lata; involucrum $1 \mathrm{~cm}$. longum, apice c. $0 \cdot 5 \mathrm{~cm}$. latum; flores lilacini; species G. crepidioidi Benth. proxima capitulis parvis confertis habitu Erechtitis insignis.

408. Cineraria Schimperi C. H. Schultz bip. (Senecio lyratipartitus ej., A. Rich. tent.). Abyssinia: Habab : Rora asgede 6-8000 aug. 1872 frutex $3 \mathrm{~m}$. altus.

1024. Emilia sagittata (Vahl.) DC. (E. humifusa (Boj.) DC. e diagn.). In Sansibariae locis cultis siccis jun. ad nov. 1873.

424. Notonia semperviva (Forsk.) Aschs. Abyssinia: Habab 3$6000^{\prime}$ in montium tractibus sterilibus jul. ad sept. 1871. 
844. eadem. Yafir in Somalensium monlibus Alil ad all. $2000 \mathrm{~m}$. in rupium calcarearum fissuris.

Huic, cui $N$. abyssinica A. Rich. certissime congener, perperam corollae flavae tribuunlur vel a viris Bentham et Hooker fil. gen. pl. II. 1, p. 446 ; sunt enim revora lilacinae.

18. Senecio leucanthemifolius Poir., Boiss.! Fl. or. III. ined., ex quo S. vernalis W. et K. var. (S. coronopifolius Aschs.! in Hildebr. pl. exs. non Desf.). In deserto Ramle mart. 1872.

63. S. coronopifolius Desf. Suez inter Trifolia culta apr. 1872. 460. S. abyssinicus C. H. Schultz bip., A. Rich. tent. (ind. S. bellidifolio ej.). Abyssinia 1872.

401. S. petitianus A. Rich. e descr. Abyssinia: Habab ad $6000^{\prime}$ aug. 1872 ; frutex allit. $4 \mathrm{~m}$.

S. tuberosus C. H. Schultz bip., A. Rich. tent. adest in coll. beccariana e Keren jul. 1870 lectus sub n. 189.

16. Calendula arvensis L. Ramle mart. 1872. apr. 1872.

60. Volutarella Lippii (L.) Cass. Inter Trifolia culta prope Suez

61. Centaurea aegyptiaca L. cum praecedente.

20. C. glomerata Vahl. Ramle mart. 1872.

21. C. dimorpha Viv. (C. eriocephala Boiss.) var. cum praecedente. Materiam possidemus ditem speciei hujusce Alexandriae martio 1824 ab Ehrenbergio lectam, quacum convenit florum structura, sed folia aliam prae se ferunt faciem; in nostra laciniae laterales abbreviatae ut terminalis minuta non distinctae, sed altera ad alteram utrinque late decurrit, at nostram quoque huc spectare nullus dubito.

423. Dicoma tomentosum Cass. Abyssinia: Habab: Bogos in planitiebus sterilibus tempore pluvioso sept. 1872. apr. 1872.

62. Cichorium divaricatum Schousb. Suez inter Trifolia culta

19. Hyoseris lucida L. Ramle mart. 1872.

17. Rhagadiolus hedypnoides All. (Hedypnois globulifera Lam.) cum praecedente.

426. Lactuca petitiana A. Rich. Abyssinia: Habab alt. 4-6000' sept. 1872.

Hujus syn. est Microrhynchus octophyllus Hochst. a Schweinf. Beitr. p. 160 lapsu ad sequentem ductum.

132. L. goreensis (Lam.) C. H. Schultz bip. Geddah apr. $18 \tilde{r} 2$.

1025. eadem. Insula Sansibar ad rudera in oppido nov. 1873 ab Europaeis interdum ut $L$. sativa $L$. comeditur; planta biennis.

844 a. Heterachaena massaviensis Fresen. E. Somalensium montibus Ahl ad altit. $1000 \mathrm{~m}$. solitaria in locis umbrosis mart. 1873 .

69. Reichardia tingitana (L.) Rth. Suez inter Trifolia apr. 1872. sept. 1872.

425. eadem. Abyssinia: Habab $3-5000^{\prime}$ tempore pluvioso aug.

1026. Sonchus Bipontini Aschs. in Schweinf. Beitr. p. 286. (S. lactucoides C. H. Schultz bip., A. Rich. tent. non Bunge). Insula Sansibar in pratis siccis herbaceis sept. 1873; radix stolonifera. 
1026. Microrhynchus nudicaulis (L.) Less. (Rhabdotheca chondrilloides Aschs.! in Hildebr. pl. exs. non. Schultz bip.). Inter Trifolia prope Suez apr. 1872.

1028. M. sarmentosus (Willd.) DC. In Sansibariae littore arenoso nov. 1873 ; rami prostrati.

131. M. glomeratus (Sieb.) Benth. et Hook. f. (Lomatolepis $\mathrm{g}$. Cass.) Geddah apr. 1872.

764. idem. Hodeida in locis humidis jun. 1872.

129-130. M. chondrilloides (Desf.) Benth. et Hook. f. Geddah apr. 1872 .

\title{
(Continuabitur.)
}

\section{Aufzählung der in der Umgebung von Pola wachsen- den Pflanzen.}

\author{
Yon Prof. Leo Neugebauer.
}

(Schluss.)

Salicornia herbacea L. V. Fisella; selten. 637.

- fruticosa L. Felsiger Seestrand (V. Vergarola, V. Ovina). 642.

Polycnemum arvense L. Brachfelder (am M. Rizzi, Stoja Musil). 698. Chenopodium urbicum L. Strassenränder (Strasse nach Policarpo und Veruda). 660.

- murale L. Schutt (M. Zaro), eine Halophytenform am Strande (V. d. Fora). 284.

- album L. Schutt. 405.

- polyspermum L. Trockene Stellen des Pra grande; sehr selten. 516 .

Beta vulgaris L. Strassenränđer. 641.

- maritima L. Schutt (hinterm Spital), F. Marina. 224, 462.

Camphorosma monspeliaca L. Humusboden nahe der Küste (V. Fisella, Stoja Musil). 590.

Halimus portulacoides Wallr. Sumpfige Buchten (V. Fisella). 639. Atriplex hortensis L. Kulturboden (Pra grande, M. Signole). 708.

- latifolia Wahlb. Raine. 703.

- rosea L. Seestrand, in unendlich vielen Formen. 702, 659.

Rumex conglomeratus Murr. Kaiserwald. 337, 440.

- pulcher L. Raine. 315.

- crispus L. Stoja Musil. 391.

- Acetosella L. Raine (M. Rizzi). 389.

Polygonum Persicaria L. Gräben (Pra grande, Stoja Musil). 716, 431.

- aviculare L. Wege, Steinbrüche. 418, ว20.

- dumetorum L. Hecken, Aecker. 586.

- Fagopyrum L. Kulturboden (am M. Signole, Anlagen vor der Schwimmschule). 567. 